\title{
Observation of a bright coronal downflow by SOHO/EIT
}

\author{
D. Tripathi ${ }^{1, \star}$, S. K. Solanki ${ }^{1}$, R. Schwenn ${ }^{1}$, V. Bothmer ${ }^{2}$, M. Mierla ${ }^{1}$, and G. Stenborg ${ }^{3}$
}

\author{
1 Max-Planck-Institut für Sonnensystemforschung, 37191 Katlenburg-Lindau, Germany \\ e-mail: d.tripathi@damtp.cam.ac.uk; [solanki; schwenn; mierla]@mps.mpg.de \\ 2 Astrophysical Institute, University of Göttingen, Germany \\ e-mail: bothmer@astro.physik.uni-goettingen.de \\ ${ }^{3}$ Catholic University of America, Washington DC / NASA Goddard Space Flight Center, Greenbelt, MD, USA \\ e-mail: stenborg@kreutz.nascom.nasa.gov
}

Received 18 July 2005 / Accepted 25 November 2005

\begin{abstract}
A distinct coronal downflow has been discovered in the course of a prominence eruption associated coronal mass ejection (CME) imaged by EIT (Extreme ultraviolet Imaging Telescope) and LASCO (Large Angle Spectrometric Coronagraph) on board SOHO (Solar and Heliospheric Observatory) on 5-Mar.-2000. Evolution of the prominences seen by EIT was tracked into the LASCO/C2 and C3 field-of-view where they developed as the core of a typical three-part CME. In contrast to the inflow structures reported earlier in the literatures, which were dark and were interpreted as plasma voids moving down, the downflow reported here was bright. The downflow, which was only seen in EIT FOV had an onset time that coincided with the deceleration phase of the core of the CME. The downflow showed a rapid acceleration followed by a strong deceleration. The downflow followed a curved path which may be explained by material following the apex of a contracting magnetic loop sliding down along other field lines, although other explanations are also possible. Irrespective of the detailed geometry, this observation provides support for the pinching off of the field lines drawn-out by the erupting prominences and the contraction of the arcade formed by the reconnection.
\end{abstract}

Key words. Sun: corona - Sun: coronal mass ejections (CMEs) - Sun: prominences - Sun: filaments

\section{Introduction}

Observations of downflows above large-scale post-flare arcades (supra-arcades) were first reported by McKenzie \& Hudson (1999) on the basis of soft X-ray observations. These downflows manifest themselves as dark structures moving through the corona towards the Sun with speeds in the range $45-500 \mathrm{~km} \mathrm{~s}^{-1}$. All events with sunward plasma motions were associated with coronal mass ejections (CMEs). One very simple explanations of these downflows could be that some portion of the CME material fails to achieve the escape velocity and consequently falls back towards the Sun under the force of the Sun's gravitational field. In that case the downflowing hot material eventually cools down and becomes visible in lines such as $\mathrm{H} \alpha$. Material falling back due to the Sun's gravitational field is termed as "coronal rain", originally based on H $\alpha$ observations (Tandberg-Hanssen 1974, p. 29). The physical explanation generally given for coronal rain is that the plasma which was evaporated into the tops of flaring loops (Forbes \& Malherbe 1986; Svestka 1998) radiates away its

* Present address: Department of Applied Mathematics and Theoretical Physics, Center for Mathematical Sciences, University of Cambridge, Wilberforce Road, CB3 0WA, UK. energy and cools down. Since no cooler counterpart was detected in $\mathrm{H} \alpha$ and at EUV wavelengths for the downflows observed in SXRs (Soft X-rays), McKenzie \& Hudson (1999) and McKenzie (2000) interpreted these downflows as plasma voids with low densities and high temperatures. These "voids" were thought to be the direct observational evidence for magnetic reconnection processes which had occurred higher up in the corona.

White-light features moving towards the Sun's surface after CME lift-off have first been detected by Wang et al. (1999) and Sheeley \& Wang (2002) based on LASCO/C2 observations. These coronal downflows occurred at altitudes of $3-5 R_{\odot}$ with speeds in the range of $50-200 \mathrm{~km} \mathrm{~s}^{-1}$. The inward moving features in white-light were observed as dark trails behind barely visible density enhancements with a cusp-like appearance. These features were interpreted as observational signatures of the closing down of magnetic field lines which were dragged outward by CMEs, providing plausible evidence for the occurrence of magnetic reconnection processes.

Recently, Innes et al. (2003a,b) reported similar features detected in EUV by the Transition Region And Coronal Explorer (TRACE; Handy et al. 1999) and SOHO/Solar Ultraviolet Measurements of Emitted Radiation (SUMER; 
Wilhelm et al. 1995). Here the downflow was observed as a dark trail of plasma moving towards the Sun at $195 \AA$ in TRACE and in the Fe XXI \& UV continuum by SUMER. These downflows were not seen in C II and Fe XII lines. Based on these observations, Innes et al. concluded that the dark trails of the plasma are likely to be the plasm avoids introduced by McKenzie \& Hudson (1999). Using complementary observations from TRACE and RHESSI, Asai et al. (2004) identified a time correlation between non-thermal radiation in the hard X-ray spectra taken by RHESSI and the times of downflows observed by TRACE at $195 \AA$. Based on these observations Asai et al. (2004) concluded that the downflow motions occurred when large amounts of magnetic energy was released, suggesting a relation of downflows with reconnection outflows.

In this paper we present an observation of a distinct downflow observed by SOHO/EIT at $195 \AA$ on 5-Mar.-2000. The downflow was observed above post-eruptive arcades (PEAs) $\sim 90 \mathrm{~min}$ after the eruption. The formation of a post-eruptive arcade observed on the disk was probably related to shrinking magnetic field lines as a consequence of magnetic reconnection after the CME eruption (Kopp \& Pneuman 1976). The data set clearly showed material flowing downward at heights below $0.5 R_{\odot}$ above the solar surface. Contrary to the earlier observations of dark downflows by SXT, TRACE and LASCO, the downflow observed in this event at $195 \AA$ was bright. In this paper we present the evolution of the prominence, associated $\mathrm{CME}$ as well as the plasma downflow.

\section{Observations}

The eruption of two large prominences marked with P1 and P2 in Fig. 1, and a barely discernible prominence marked with P3 was recorded on 5-Mar.-2000 by EIT at $195 \AA$. The sequence of the running difference images in Fig. 1 shows the almost simultaneous eruption of $\mathrm{P} 1, \mathrm{P} 2$ and $\mathrm{P} 3$. The running difference images reveal the proper motion of the features in the direction of motion (Sheeley et al. 1999). The formation of a PEA starts at $\approx 16: 12$ UT underneath P1. The PEAs evolve in height with time as can be seen in Fig. 1 and also in Fig. 3.

The associated CME was first seen at 16:54 UT in the LASCO/C2 field-of-view (FOV), (see Fig. 2). The CME later, after 17:06 UT, exhibited the typical three part structure, being comprised of a bright front, a dark cavity and a bright core. Possible individual CMEs associated with prominence eruptions P1, P2 and P3 cannot be uniquely distinguished in the coronagraphic images. The prominences $\mathrm{P} 1$ and $\mathrm{P} 2$ were later seen as the core of the CME at 17:06 UT. The prominences P1 and P2 are clearly discernible until 17:54 UT in LASCO/C2 observations marked in the middle right panel of Fig. 2. Later on at 18:06 UT, only P1 can be uniquely distinguished.

At 17:36 UT, i.e., $90 \mathrm{~min}$ after the onset of the eruption, a bright sunward moving feature, hereinafter referred to as the downflow, was detected by EIT at $195 \AA$ A. Figure 3 displays the running difference images taken by EIT at $195 \AA$ starting from the first detection (top left corner image) of the downflow until the time it vanished (bottom right corner image). The downflow is visible as the bright dot at the top edge of the top left image of Fig. 3. With passing time the bright structure

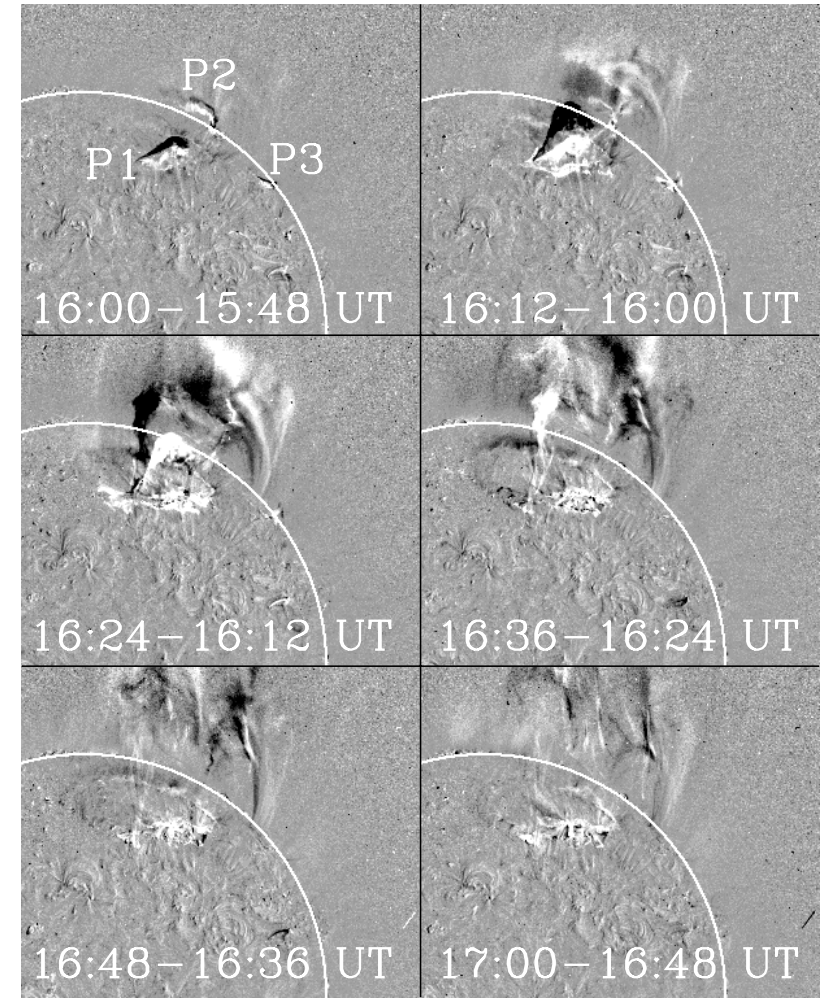

Fig. 1. Sequence of running difference images taken by SOHO's Extreme-ultraviolet Imaging Telescope (EIT) at $195 \AA$ on 5-Mar.2000. The images show the simultaneous eruption of three prominences marked as P1, P2 and P3. In the images North is pointing upwards and West towards the right.

extended downward, branching into two streams during the process. At least the right branch did not follow a straight or even regularly curved path, but showed a kink, clearly seen in the difference images at 18:24 UT. Since coronal plasma cannot cross magnetic field lines, it is either following a kinked field line or bright downward moving plasma is outlining the loops that the plasma is sliding down along. According to Fig. 3 the left branch of the downflow seemed to be linked to the eastern foot-point of P1 whereas the right branch seemed to be headed towards P3. Since we have no Doppler shift measurements we cannot rule out that we are only observing a brightening front moving downward, without any associated material flow.

The images taken by EIT and LASCO were further processed in order to enhance the contrast of fine-scale features by applying a wavelet decomposition technique established by Stenborg \& Cobelli (2003) which is based on the work of Fligge \& Solanki (1997). This technique involves multiple applications of the wavelet transform and corresponds to a wavelet packet analysis, which leads to a better separation between signal and noise, thus allowing structure with finer details to be processed. The algorithm used in the processing of the images was the 2D-à trous transform. Most of the noise in the image is concentrated in the high frequency scale in the wavelet plane. A weighted reconstruction scheme is employed in which during the process of reconstruction considerably less weight can be given to the scale containing noise. More details about the algorithm can be found in Stenborg \& Cobelli (2003). 


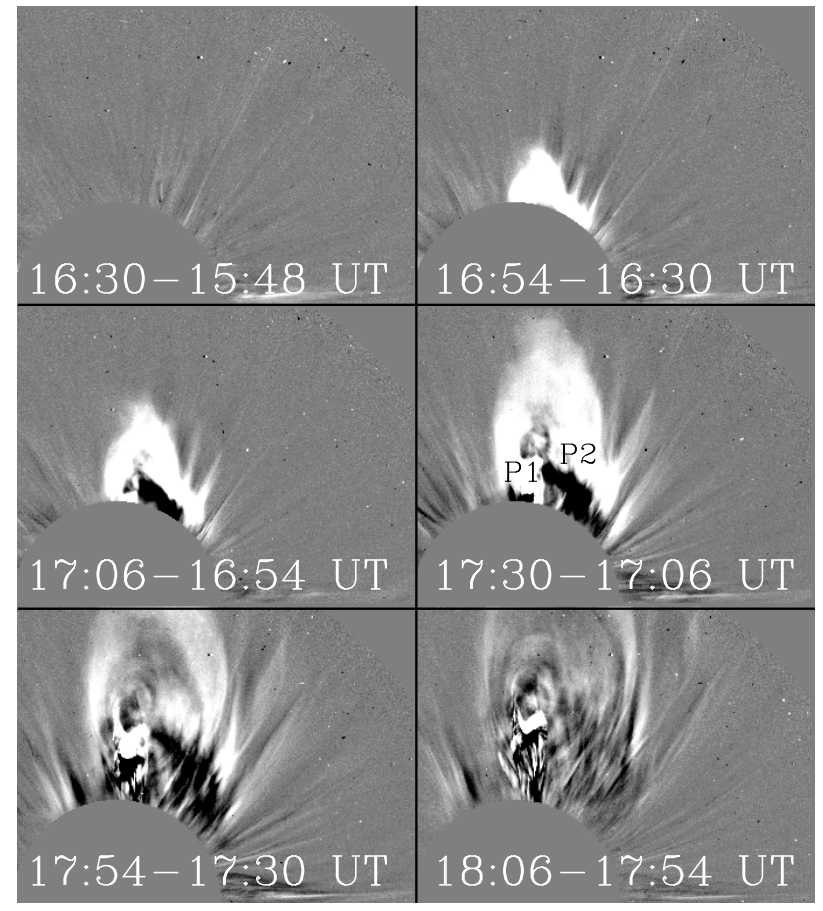

Fig. 2. Series of running difference images showing the evolution of a white-light CME detected by the LASCO/C2 coronagraph on 5-Mar.2000. The CME followed the prominence eruptions shown in Fig 1. The first appearance of the CME in the LASCO FOV is shown in the upper middle image. In later images the leading edge, dark cavity and bright core of a typical three-part CME can be seen. In the middle right image $\mathrm{P} 1, \mathrm{P} 2$ mark the prominence material.

The left panel of Fig. 4 displays the stack plot of the strips of the solar corona cropped from the wavelet processed EIT images. The figure was produced by cutting an arch-shaped strip of the solar corona above the limb (see Fig. 4, right panel) for the position angle (PA) between $290^{\circ}$ and $360^{\circ}$. The PA was measured starting from the north pole in counterclockwise direction as illustrated in the right panel of Fig. 4. A bi-linear interpolation was performed in order to transform the arch-shaped images into a rectangular shape. The eruption that started at $\sim 15: 48$ UT is best visible at 16:24 UT (see "d") when a prominence is in the center of the FOV. By 17:24 UT ("i") the entire material had passed out of the EIT FOV. No visible plasma-motion associated with the erupted features are seen in images "i" to " $k$ ", although careful inspection of frame " $k$ " reveals a faint brightening at the top edge which is the first sign of the downflowing material. At 18:00 UT ("l") and very clearly at 18:12 UT ("m") plasma moving towards the Sun's surface was detected. The path of the downflowing plasma suggests that it was channeled by the magnetic field configuration of the corona (see images from " $\mathrm{m}$ " to " $\mathrm{q}$ "). Interestingly, in frames "g" and "h" a bright filamentary structure is seen at a similar location as one branch of the downflowing material approximately an hour later.

There was no clear evidence for a downflow of plasma in white-light images taken by LASCO/C2. In order to verify this, we produced height-time diagrams using running difference images taken by EIT at $195 \AA$ and LASCO/C2 at a specified

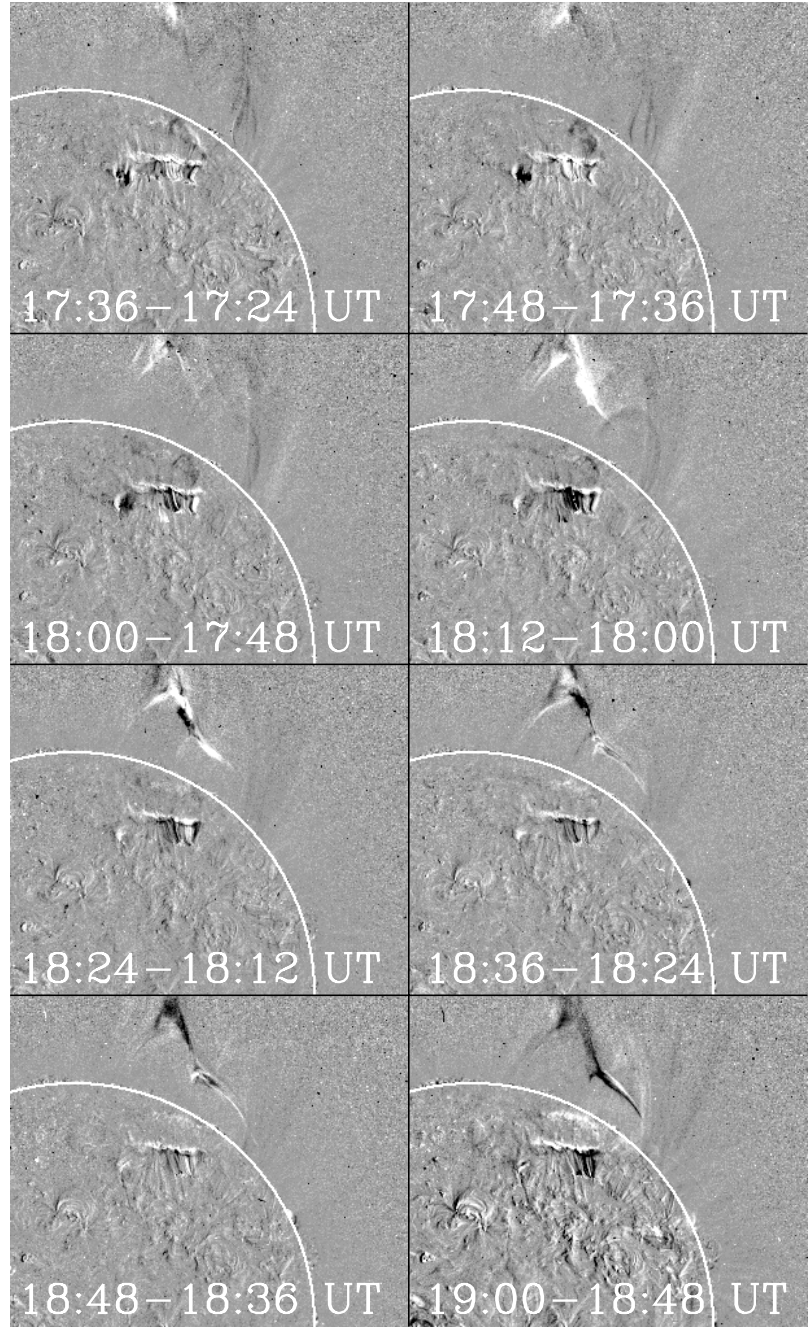

Fig. 3. Series of running difference images taken by EIT at $195 \AA$ showing the morphology of the sunward moving material (downflow) on 5-Mar.-2000.

position angle (PA). Figure 5 shows the height-time diagrams for LASCO/C2 (upper panel, $\mathrm{PA}=347^{\circ}$ ) and EIT (lower panel, $\mathrm{PA}=345^{\circ}$ ). The height-time diagrams were produced by cropping a thin strip at the specified position angle, starting above the limb at $1.02 R_{\odot}$ in the case of EIT and starting above the occulter at $2 R_{\odot}$ in the case of LASCO/C2. The strips extended to $1.36 R_{\odot}$ for EIT and to $6.0 R_{\odot}$ for $\mathrm{LASCO} / \mathrm{C} 2$. In the lower panel (height-time diagrams for EIT) of Fig. 5, both the outflow as well as the downflow were detected. They are marked by arrows. Whereas the downflow can be easily recognized by the fact that the bright structure moves down as time progresses, the outflow is so rapid that it appears as a vertical streak. The EIT height-time diagram allows us to measure the speed of the downflow by following the trajectory along the boundary of the moving dark and bright features. The downflow speed obtained at the position angle of $345^{\circ}$ was $25 \mathrm{~km} \mathrm{~s}^{-1}$. The bottom panel of Fig. 5 reveals that the downflow first became visible at the height of $1.36 R_{\odot}$, i.e., at the edge of the FOV and was seen until the height of $1.24 R_{\odot}$ at this particular position angle. In the upper panel of Fig. 5 (height-time diagram for LASCO/C2) only plasma outflow could be definitely identified. A specific 

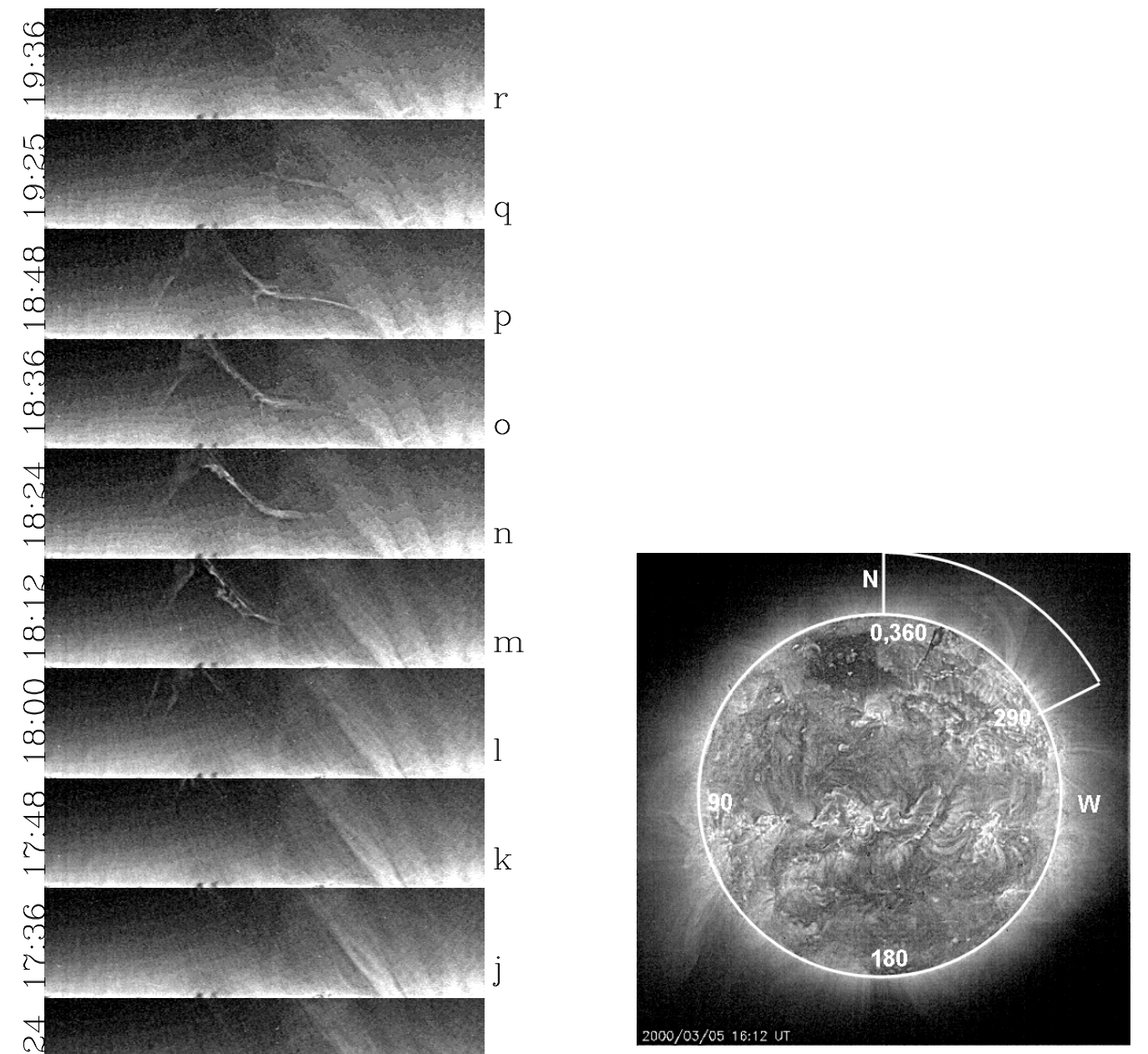

Fig. 4. Left panel: stack plot of cropped strips of the solar corona above the limb in EIT images. Time is running from bottom to top. The frames start at 15:48 UT ("a") and finishes at 19:36 UT ("r"). Each frame corresponds to the arch shaped strip marked in the right panel. The right corner denotes the position angle $290^{\circ}$ and the left denotes $360^{\circ}$. Right panel: wavelet processed EIT image taken on 5-Mar.-2000 at 16:12 UT. The area marked with the arch-shaped strip is shown in the left panel. The convention for measuring position angle is marked.
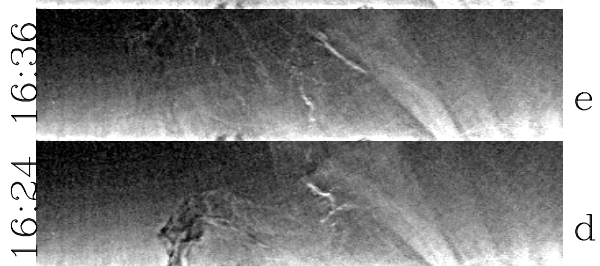

d

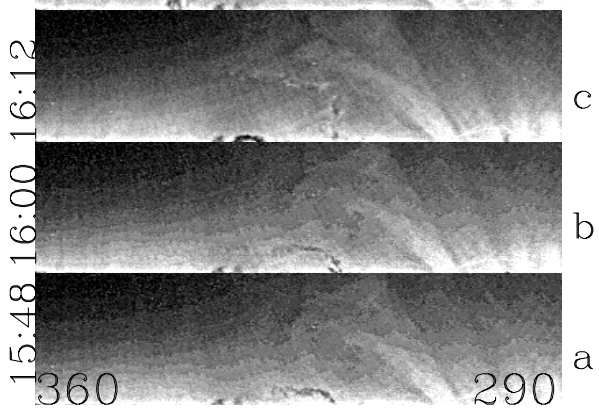



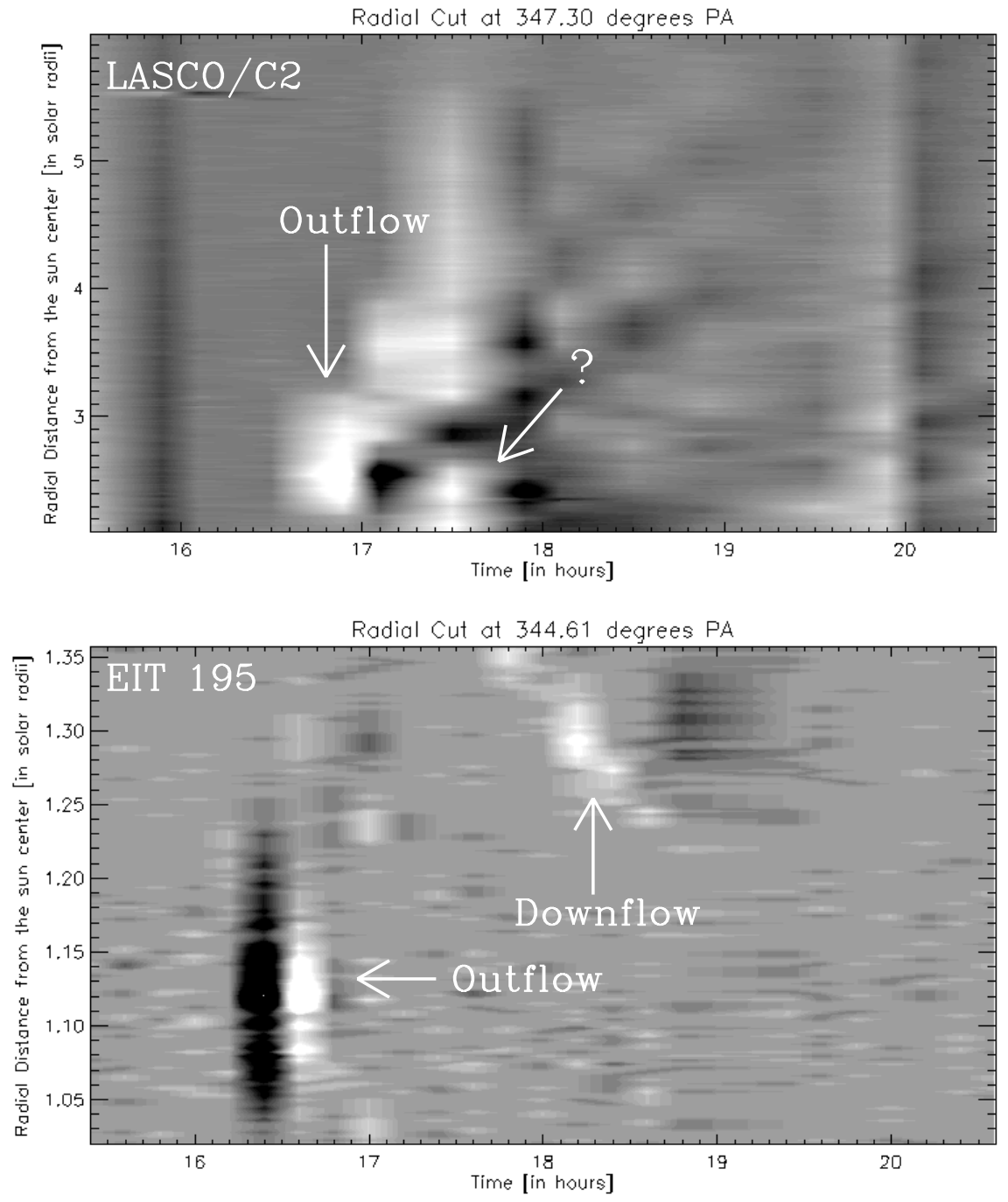

Fig. 5. Top panel: height-time diagram for LASCO/C2 running difference images at position angle $347^{\circ}$ from 15:00-20:30 UT. Bottom panel: height-time diagram for EIT $195 \AA$ A running difference images at position angle $345^{\circ}$. Note that the position angles are measured starting from solar north in counter clock wise direction as shown in Fig. 4. Outflows and downflow of the coronal material are labeled.

white-light feature in the height-time diagram of LASCO/C2, whose behavior could not be uniquely interpreted, is marked by the question mark in the upper panel. It may conceivably be the start of a downflow, but the backward extrapolation of the trajectory of the downflow seen in EIT does not match with this feature in time. Also, no outward motion of this feature in the height-time diagram was seen in $\mathrm{LASCO} / \mathrm{C} 2$. Because of the lack of the observation from 1.35 to $2.0 R_{\odot}$, it becomes difficult to make any substantial conclusion about the feature marked with the question mark.

\section{Evolution of prominence, $\mathrm{CME}$ and downflow}

The wavelet sharpening of EIT and LASCO/C2 and C3 coronagraphic images provided an opportunity to track the same feature in the FOV of different instruments. Figure 6 displays the measurements performed of the prominence in EIT, and of the core and leading edge of the CME in the LASCO/C2 and C3 FOV based on the wavelet processed images. The asterisks in the figure reflect the data points obtained for the evolution of the prominence in the EIT FOV, the diamonds and triangles represent the data points obtained for the core of the CME in the LASCO/C2 and C3 FOV shown in Fig. 6. The crosses represent the leading edge of the $\mathrm{CME}$ based on LASCO/C2 and $\mathrm{C} 3$ observations. The leading edge was not identified in the EIT images because either the loops were already initially large, lying outside the EIT FOV before the CME erupted or the plasma in the loops did not radiate at $195 \AA$. The solid dots represent the data points obtained for the downflow seen in the EIT FOV. They refer to the right branch of the downflow which we analyze in detail. For the other branch the reliability of the speed determination was lower due to a higher speed $\left(>\sim 140 \mathrm{~km} \mathrm{~s}^{-1}\right)$.

In Fig. 6, the top panel shows the variation of the position angle of the tracked features with time. The similar variation in the position angle of the prominence in EIT, and the CME core in $\mathrm{C} 2$ and $\mathrm{C} 3$ makes the assumption more plausible that we tracked the same features in the FOV of the different instruments. As can be seen in the plot, the prominence was deflected by an angle of about $\sim 15^{\circ}$ in the early phase of its evolution. Although we do not have any measurement of the leading edge 

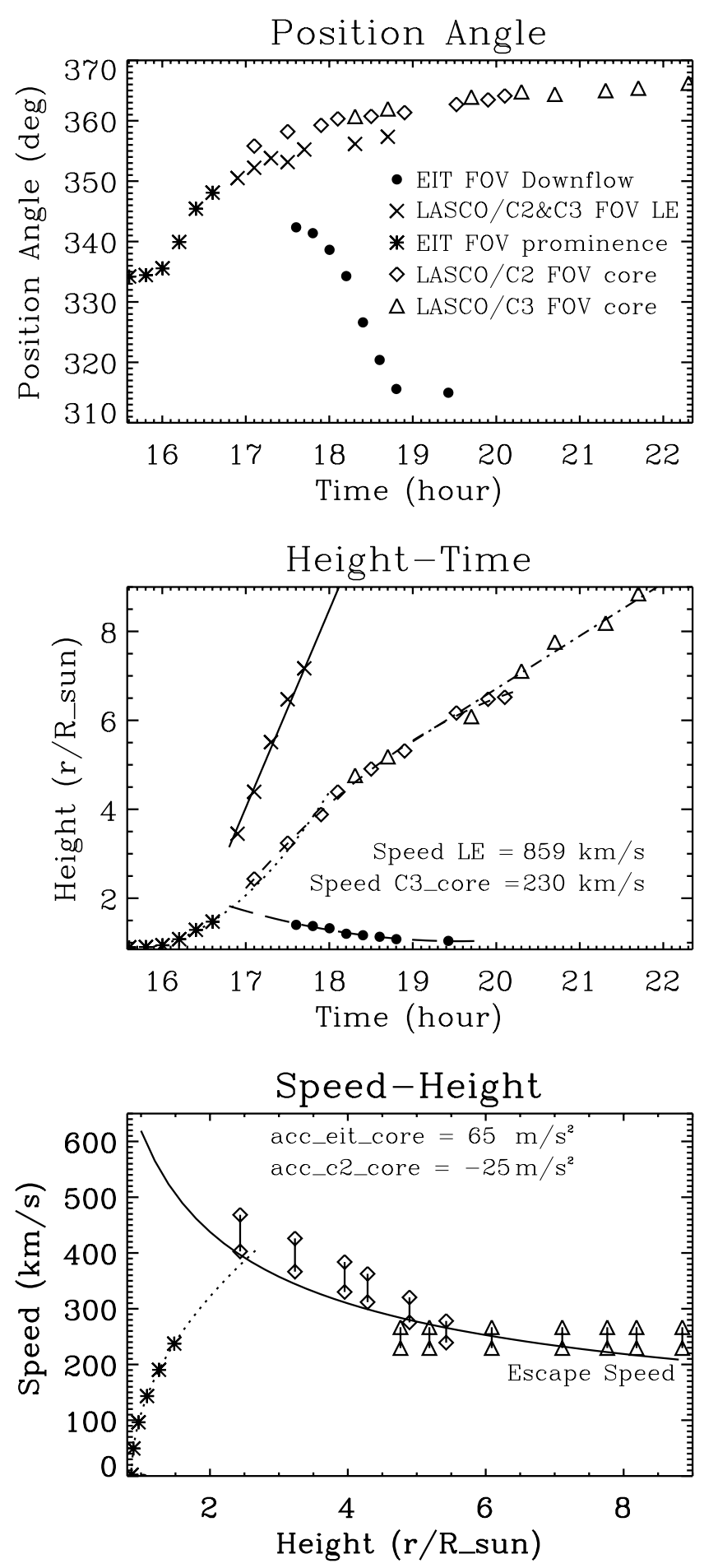

Fig. 6. Top panel: position angle variation of the prominence in the EIT FOV (asterisks), the CME core in LASCO/C2 (diamonds), the $\mathrm{CME}$ core in LASCO/C3 (triangles), the leading edge (crosses) in LASCO/C2 and $\mathrm{C} 3$ and the downflow in EIT FOV (dots). Middle panel: height-time profile for the prominence, the core, the leading edge and the downflow. The solid and dashed-dotted lines are best linear fits to the data points. Other lines are the best quadratic fits to the data points. Bottom panel: the speed-height profiles of the features mentioned above. Bottom points of bars represent the speeds projected in the sky plane and top points represent the corrected speeds based on the source region location. The speeds projected in the sky plane were derived from the polynomial fits to the height-time data in the middle panel. The solid line represents the escape speed profile. during the early phase of evolution, the trend shown by the position angle in the range of 2 to $4 R_{\odot}$ reveals that it also passed through a similar deflection phase as the prominence and core of the CME. The deflection observed in the prominence and the leading edge of the CME is comparable to the results derived in a systematic study by Cremades \& Bothmer (2004). The downflow seems to have started at position angle $340^{\circ}-345^{\circ}$. The position angle profile of the downflow reflects the complex motion of the downflow seen in Figs. 3 and 4.

The middle panel represents the height-time profile of the leading edge, prominence, the core of the CME and the downflow. The different symbols have the same meaning as in the top panel of Fig. 6. The solid line is a linear fit to the data points for the leading edge of the CME, yielding a constant speed of $860 \mathrm{~km} \mathrm{~s}^{-1}$ (projected on the plane of the sky) in the LASCO/C2 and C3 FOV. The dotted line is the second order polynomial fit to the data points obtained for the prominence in the EIT FOV. The dashed line is the second order polynomial fit to the data points obtained for the core of the CME based on LASCO/C2 observations and the dashed-dotted line is the linear fit to the data points for the core of the CME based on the LASCO/C3 observations. The overlap in the data points of $\mathrm{LASCO} / \mathrm{C} 2$ and $\mathrm{C} 3$ makes the assumption plausible that the same feature was tracked in the $\mathrm{C} 2$ and the $\mathrm{C} 3 \mathrm{FOV}$. The long dashed line is the second order polynomial fit to the data points obtained for the downflow based on the EIT observations. The height-time profile of the outflowing plasma in the EIT FOV shows a positive curvature suggesting that the prominence passed through an acceleration process in the early phase of its evolution in agreement with the conclusion reached by Webb \& Jackson (1981). The measured acceleration was about $65 \mathrm{~m} \mathrm{~s}^{-2}$. Furthermore in the LASCO/C2 FOV it shows a negative curvature, suggesting a deceleration by about $25 \mathrm{~m} \mathrm{~s}^{-2}$. Finally, the height-time profile of the core of the CME becomes nearly a straight line corresponding to a nearly constant speed of $230 \mathrm{~km} \mathrm{~s}^{-1}$ higher up in the corona. Note that there was a big difference in the speed of the core (associated prominence) and the leading edge of the CME, which travelled with a nearly constant speed of $860 \mathrm{~km} \mathrm{~s}^{-1}$ during the whole time that it was observed.

The height-time profile of the downflow (middle panel of Fig. 6), by a simple extrapolation back in time, intersects the height-time profile of the outflow at a height of about $1.8 R_{\odot}$ during its deceleration phase. Therefore, possibly the downward moving plasma is a part of the outward moving plasma seen earlier by EIT and the downflow started during the deceleration phase of the outflow in the height range $1.5-1.8 R_{\odot}$.

The bottom panel of Fig. 6 displays the speed-height profile of the outflow. The speeds of the outflow were derived from the fitted height-time polynomials in the middle panel and then were corrected based on the source region location which yielded $14 \%$ larger speeds. Both the speeds derived from LASCO are represented in the plot, joined by bars. The bars upper points represent the corrected speed. The symbols have the same meaning as in the top panel of Fig. 6. The solid line is the escape speed profile calculated for a ballistic body, i.e. taking into account only the gravitational acceleration. By a simple extrapolation, the speed-height profile of the filament intersects 


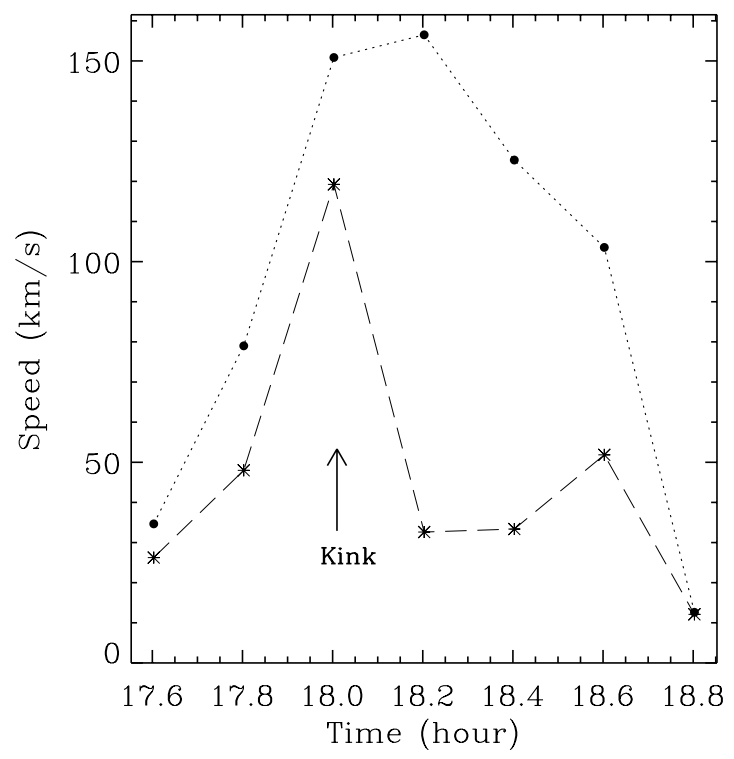

Fig. 7. Projected absolute (dotted line) and vertical (dashed line) speed profiles for the observed downflow. The arrow marks the time of the kink in the downflow's trajectory based on the EIT observations.

the escape speed profile at $\sim 2.5 R_{\odot}$ within uncertainties of the measured speeds of the core of the CME appears to be moving roughly with escape speed above this level.

Finally, in Fig. 7 we plot the absolute (dotted) and radial (dashed) speeds of the downflow projected on to the plane of the sky as a function of time. The arrow marks the time when the kink in the right branch of the downflow path was detected based on EIT observations (see Fig. 3). The downflow shows a rapid acceleration until it arrives at the kink location. Thereafter it starts to decelerate. Initially the downflowing gas is accelerated in both horizontal and vertical directions by about a quarter of the solar gravitational acceleration $\left(61-100 \mathrm{~m} \mathrm{~s}^{-2}\right.$ for the first two points shown in Fig. 7). Hence, the downflow does not correspond to freely falling gas. After the kink the downflow decelerates most strongly in the vertical direction.

\section{Comparison with Yohkoh-SXT observations}

Figure 8 displays portions of the images recorded on 5-Mar.2000 by the soft X-ray telescope (SXT; Tsuneta et al. 1991) aboard Yohkoh through the Al-Mg filter. The specific timings of the respective frames are indicated in the figure. The post-eruptive arcade corresponding to the locations of erupting prominences P1 and P2 (shown in Fig. 3) is clearly seen. Below the location of the downflow the SXT loops have somewhat pointed apex reminiscent of the structure often seen in Yohkoh images just after flares.

In order to compare the spatial location of the feature seen in EIT and SXT images, we display in Fig. 9 an EIT image co-aligned with an SXT image recorded very close in time (EIT: 18:12 UT, SXT: 18:11 UT). The EIT image is shown in the background and the SXT image is overlayed by invoking a transparency of about 50 percent in the SXT image. From the figure it is evident that the downflow seen in EIT is coming down near the top (near the cusp often seen in post flare
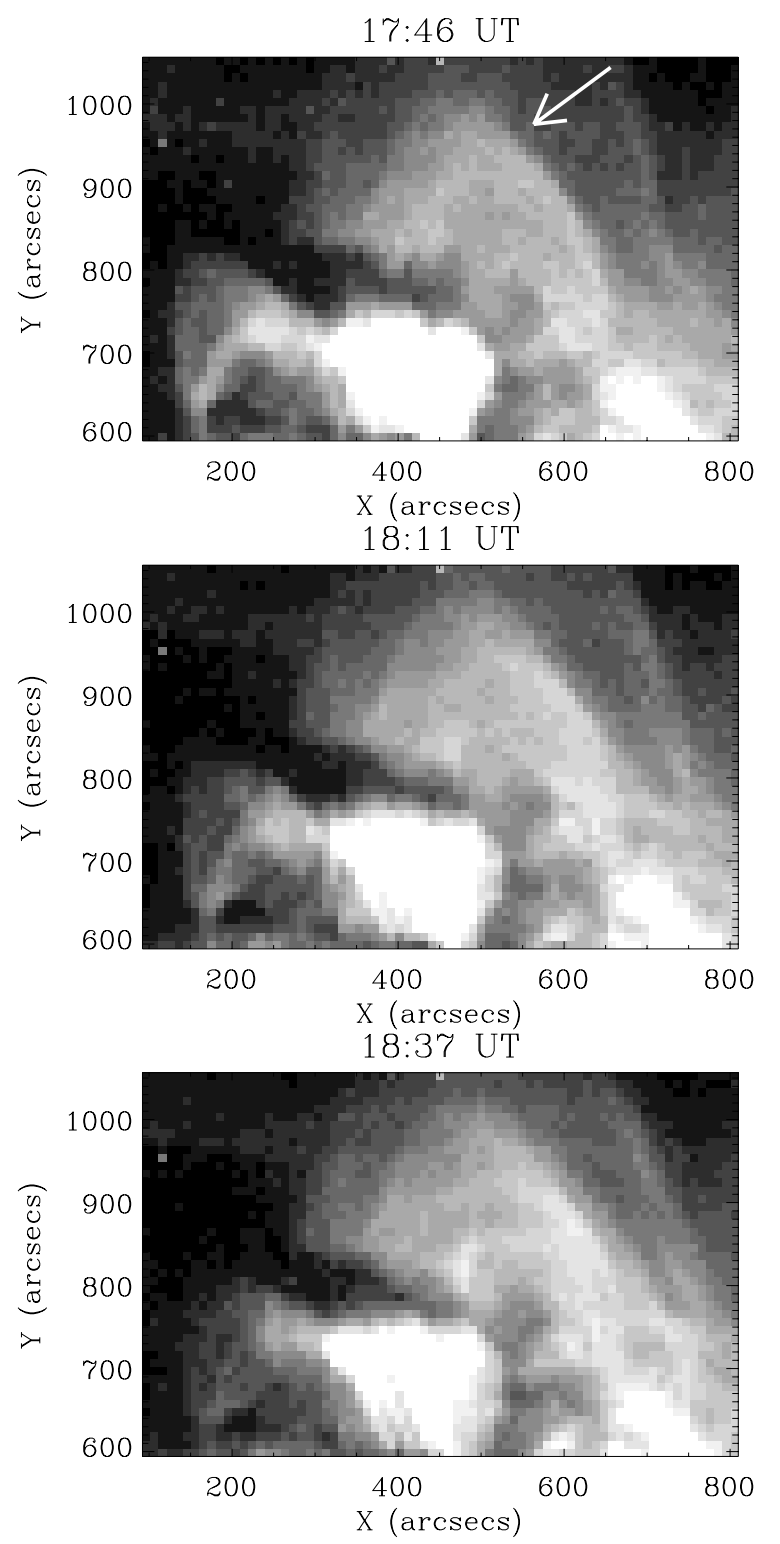

Fig. 8. Images taken by the soft $x$-ray telescope (SXT) through the Al-Mg filter aboard Yohkoh on 5-Mar.-2000 showing cusp-shape feature at the location of the downflow. Timing of each frame is labelled. The arrow in the top panel demarks the location of the downflow.

loops or arcades) of a set of post flare loops. These loops are only seen in the SXT images since they are still too hot to be recorded in the EIT $195 \AA$ bandpass. In SXT images these loops were present already before the downflowing material first became visible to EIT. The material then moves down along the right leg of this loop system (at least this is true for the right branch of the downflow; for the left branch there seems to be very little $\mathrm{X}$-ray emission).

\section{Discussion and conclusions}

A downward moving brightening, which we refer to in short as the downflow, was observed above post-eruptive arcades by the Extreme-ultraviolet Imaging Telescope (EIT) at $195 \AA$. This is the first time to our knowledge that a bright downflow has been 


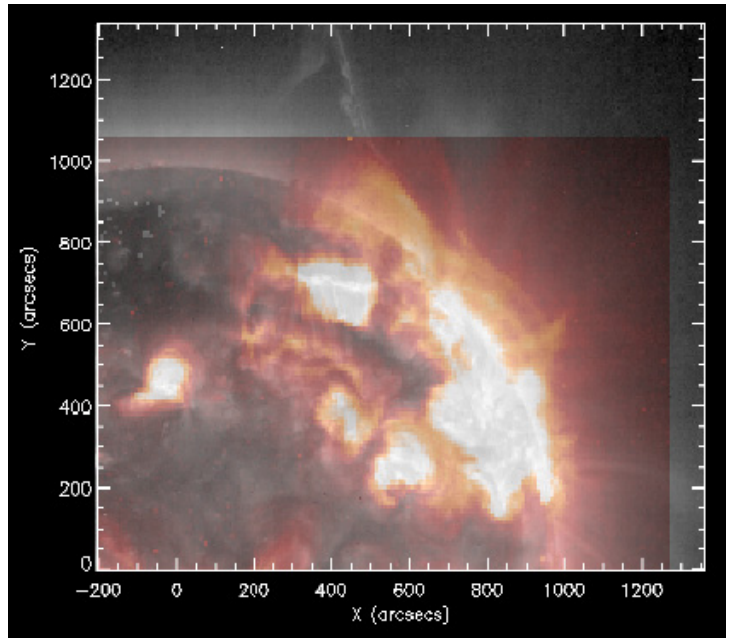

Fig. 9. Co-aligned over-plotted EIT (background) and SXT images (foreground) taken at 18:12 and 18:11 UT respectively. Note that the right branch of the EIT downflow is directed to the top of the cusp seen in the SXT image.

observed at coronal temperatures. Previously observed downflows have all been dark and have been interpreted as voids. This explanation obviously does not apply to the current observations. The downflow was observed about $90 \mathrm{~min}$ after the onset of a prominence eruption (marked P1 in Fig. 1). As seen in EIT the prominence started to erupt at around 16:00 UT and was later observed as the core of a CME after 17:06 UT by the LASCO/C2 coronagraph in white-light. Detailed analysis of the images revealed that:

1. A deflection of $\sim 15^{\circ}$ towards the north pole was seen in the early phase of the prominence (P1) eruption.

2. The prominence (P1) first accelerated with a constant acceleration of about $65-70 \mathrm{~m} \mathrm{~s}^{-2}$ in the EIT FOV $\left(1.5 R_{\odot}\right)$ until it reached escape speed, then decelerated (at approximately $25 \mathrm{~m} \mathrm{~s}^{-2}$ in the LASCO/C2 FOV (2-5 $\left.R_{\odot}\right)$ ) following roughly the escape speed curve, suggestive of a ballistic trajectory. The prominence is identified with the core of the CME seen by LASCO/C2 and C3.

3. The leading edge of the CME was only observed in the LASCO/C2 and C3 FOV. The leading edge moved with a speed of $860 \mathrm{~km} \mathrm{~s}^{-1}$, which is much faster than the core of the CME.

4. The downflow was first seen $\approx 90 \mathrm{~min}$ after the eruption at a height of about $1.5 R_{\odot}$ at the edge of the EIT FOV. There was no clear evidence for downflow in $\mathrm{LASCO} / \mathrm{C} 2$ and $\mathrm{C} 3$ images.

5. The height-time profile (Fig. 6, middle panel) provides clear evidence that the downflow started during the deceleration phase of the core of the CME.

6. The downflow does not propagate vertically, but appears to follow paths outlining groups of loops. Two branches emanating from the same apex point are seen. The right branch shows a clear kink.

7. The absolute and radial projected speed of the downflow shows a rapid acceleration in its early phase for the right branch before it arrives at the location of the kink. After that it moves more slowly in a more horizontal direction. The kink corresponds to the apex of a post-eruptive arcade of hot loops visible in Yohkoh SXT images already prior to the downflow.

Let us now consider possible scenarios to explain these observations. Quite basically we need to distinguish between a downflow of material, a downflow of energy, leading to a heating of the gas and a downward propagating cooling front. In the case of heating, a source of energy release lying outside the EIT FOV would lead to a transport of energy downwards into the field of view by conduction. The brightening could occur when the ambient gas has been heated to a temperature to which EIT is sensitive. The filamentary structure of the bright downflow requires a higher density at these filament locations or enhanced conduction along field lines. A higher density may be due to gas left behind from the CME. A problem with this scenario is why the cool gas did not flow down due to gravitational attraction before it was heated up. The scale height of sub-MK gas is too low for it to contribute strongly to emission at $1.5 R_{\odot}$. The free fall time is on the order of $10^{3} \mathrm{~s}$, i.e. shorter than the time between the passage of the last outward flowing (visible) material in the EIT FOV and the appearance of the downflow.

A cooling front provides a more viable alternative. In this case the plasma is initially too hot to be well visible in the EIT $195 \AA$ channel. Loops with a temperature of 6-8 MK are regularly observed by Yohkoh (e.g. Nitta 2000) \& SUMER (e.g. Wang et al. 2003). Cooling by radiative losses propagates from regions of higher to lower density, since radiative losses are more efficient at high density $\left(\sim n_{\mathrm{e}}^{2}\right)$. Hence the downward propagation of the cooling front would require a density excess in the higher layers, which is not straight forward to achieve. One advantage of this scenario is that the kink in the "downflow" does not pose any problem, since the cooling front need not follow a field line. On the other hand, it is not clear why only very localized regions brighten at a give time and not all the material along the respective field lines, since thermal conduction should produce a relatively homogeneous temperature. Although this scenario could in principle be tested by looking for brightenings in Yohkoh SXT images prior to those in EIT, the Yohkoh SXT field-of-view did not reach sufficiently high up to allow such a test to be carried out.

Turning now to the scenario of actual downflow of plasma as the source of the downward propagating brightening, we see that the plasma cannot be in free fall. The speed profile of the downflow (see Fig. 7) shows a rapid acceleration in the early phase which finally starts to decelerate as soon as the downflow arrives at the location of the kink. Such a behaviour would be more typical of plasma accelerated by a reconnection event taking place outside the field of view of EIT (and inside the occulting disk of LASCO C2). This interpretation is supported by the fact that in the height-time diagram (middle panel of Fig. 6) the extrapolations of the trajectories of the ejected prominence material and of the downflowing material intersect at around the conjectured location of the reconnection. Another argument for reconnection taking place is that the prominence material in the EIT FOV shows significant acceleration and if extrapolated 
D. Tripathi et al.: EI

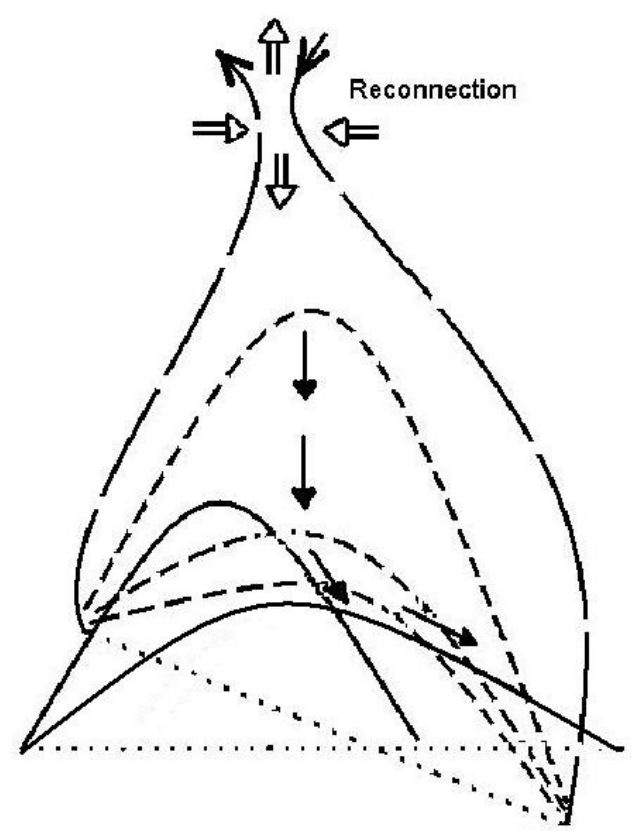

Fig. 10. Schematic illustration of the observed downflow, in particular its right branch. The long-dashed lines are magnetic field lines initially holding the prominence. The solid field lines are post-eruptive arcades and dashed-lines represent a single field line at three different times after the reconnection.

along the dotted line in the bottom panel of Fig. 6 would reach escape speed easily. Within this basic picture there are again different scenarios:

a) The plasma is flowing down freely along a field line. In this case the field line along which the right branch of the downflow moves would have to be kinked.

b) The downflow outlines the tops of an arcade of contracting loops. This model has the advantage that field lines are ejected along with rapidly flowing gas. For reconnection occurring higher up in the atmosphere, the downward accelerated field would have the right geometry.

c) A combination of both effects is also possible.

The contracting loops in scenario b) would be aligned along the line-of-sight (making their apex appear brightest due to large column density) and would be roughly perpendicular to the loops along which they are sliding down. The loops located on the left of the apex of the underlying loop move towards the left footpoint, those on the right move down towards the right footpoint, explaining the partition into two branches. The kink is located where the contracting loop stops sliding down one loop and starts sliding down another, flatter loop (i.e. with a smaller ratio between the length of the loop and the separation between its foot points) with foot-points lying further apart.

Figure 10 illustrates the magnetic field geometry associated with this scenario of the observed downflow. The longdashed lines are field lines which were initially holding the prominence. The solid lines belong to post-eruptive arcades. The dashed lines are field lines after the reconnection which are sliding down along other field lines. The arrows indicate the direction of motion of the contracting arcade (in the illustrated case corresponding to the right hand branch of the downflow).

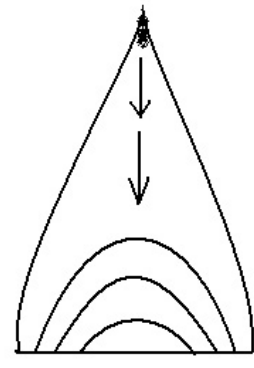

t1

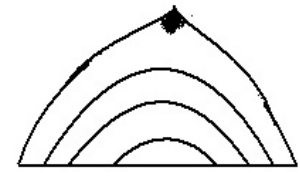

t2

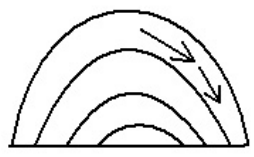

t3
Fig. 11. Schematic illustration of the downflow given by scenario c). $\mathrm{t} 1, \mathrm{t} 2$ and $\mathrm{t} 3$ demonstrate the time evolution of the downflow.

In scenario c) an initial rapid contraction takes place of a loop formed by the reconnection of oppositely directed field lines stretched out in the wake of the CME. The scenario is sketched in Fig. 11. Once the loop arrives back at a nearly circular shape, like the rest of the arcade, it stops contracting. Then the material located near its apex slides down along one of the loop's legs to its foot point, explaining the kink in the flow direction. This scenario is supported by the SXT observation that the downflowing material is indeed located above the apex of the post-eruptive arcade, which shows signs of a cusp. Since cusp-shaped structures are often discussed in context of reconnection (see e.g., Acton et al. 1992) and shrinkage of magnetic field lines (Forbes \& Acton 1996), this strengthens the point that reconnection is involved in driving the downflow.

Although scenarios b) and c) satisfy a number of observational constraints, the question remains open as to why such bright downflows are so rare that none was seen prior to the one reported here. Possibilities are:

- Magnetic reconnection after CME ejection is rare.

- Magnetic reconnection is common but usually the gas density at and below the location of reconnection is low, so that the downflow is not visible.

- The special geometry of this particular event (multiple prominences erupting simultaneously) aligns the contracting loops such that they are best visible.

In any case, we conclude that the current observations add further evidence for the occurrence of post CME reconnection and contraction of the drawn-out field lines, at least for some CMEs. It is well worth searching carefully in archival data to estimate the true rate of occurrence of such downflows.

Acknowledgements. We would like to thank an anonymous referee for his comments which improved the quality of the manuscript. DT is thankful to Dr. B. Inhester and Dr. N.-E. Raouafi for several useful discussions. This study is part of the scientific investigations of the project Stereo/Corona supported by the German "Bundesministerium für Bildung und Forschung" through the "Deutsche Zentrum für Luftund Raumfahrt e.V." (DLR, German Aerospace Center) under project number 50 OC 0005 . Stereo/Corona is a science and hardware contribution to the optical imaging package SECCHI on the NASA STEREO mission. We wish to thank the SOHO/LASCO/EIT consortium for providing the data and the software libraries and we acknowledge use of the CME catalog generated and maintained by NASA and The Catholic University of America in cooperation with the Naval 
Research Laboratory. SOHO is a project of international collaboration of ESA and NASA.

\section{References}

Acton, L. W., Feldman, U., Bruner, M. E., et al. 1992, PASJ, 44, L71 Asai, A., Yokoyama, T., Shimojo, M., \& Shibata, K. 2004, ApJ, 605, L77

Brueckner, G. E., Howard, R. A., Koomen, M. J., et al. 1995, Sol. Phys., 162, 357

Cremades, H., \& Bothmer, V. 2004, A\&A, 422, 307

Delaboudinière, J.-P., Artzner, G. E., Brunaud, J., et al. 1995, Sol. Phys., 162, 291

Fligge, M., \& Solanki, S. K. 1997, A\&A, 124, 579

Forbes, T. G., \& Acton, L. W. 1996, ApJ, 459, 330

Forbes, T. G., \& Malherbe, J. M. 1986, ApJ, 302, L67

Handy, B. N., Acton, L. W., Kankelborg, C. C., et al. 1999, Sol. Phys., 187,229

Innes, D. E., McKenzie, D. E., \& Wang, T. 2003a, Sol. Phys., 217, 247
Innes, D. E., McKenzie, D. E., \& Wang, T. 2003b, Sol. Phys., 217, 267

Kopp, R. A., \& Pneuman, G. W. 1977, Sol. Phys., 50, 85

McKenzie, D. E., \& Hudson, H. S. 1999, ApJ, 519, 93

McKenzie, D. E. 2000, Sol. Phys., 195, 381

Nitta, N. 2000, Sol. Phys., 195, 123

Sheeley, N. R., \& Wang, Y, -M. 2002, ApJ, 579, 874

Sheeley, N. R., Walters, J. H., Wang, Y.-M., \& Howard, R. A. 1999, J. Geophys. Res., 104, 24739

Stenborg, G., \& Cobelli, P. J. 2003, A\&A, 398, 1185

Svestka, Z. K., Fárník, F., Hudson, H. S., \& Hick, P. 1998, Sol. Phys., 182,179

Tandberg-Hanssen, E. 1974, Solar Prominences, 12 (DordrechtHolland/Boston-USA: D. Reidel Publishing Company), 29

Tsuneta, S., Acton, L., Bruner, M., et al. 1991, Sol. Phys., 136, 37

Wang, T. J., Solanki, S. K., Curdt, W., et al. 2003, A\&A, 406, 1105

Wang, Y.-M., Sheeley, N. R., Howard, R. A., St. Cyr, O. C., \& Simnett, G. M. 1999, Geophys. Res. Lett., 26, 1203

Webb, D. F., \& Jackson, B. V. 1981, Sol. Phys., 73, 341

Wilhelm, K., Curdt, W., Marsch, E., et al. 1995, Sol. Phys., 162, 189 